\title{
Associations between socioprovincial factors and self-reported mental disorders among students from grade 4 to 8 in rural China
}

Ming Guan ${ }^{1,2,3}$ (D)

\begin{abstract}
Background: The focus on child mental health in developing countries was increasing. However, little was known in China. This study aimed to explore the associations between socioprovincial factors and self-reported mental disorders in rural China.

Methods: Data were from a publicly available survey with 54,498 students from Grade 4 to 8 in rural China. Chisquare test was used for descriptive analysis. Self-reported mental disorders included overall mental disorder, study anxiety, personal anxiety, loneliness, guilt, sensitivity, symptomatic psychosis, phobia, and impulsivity. Multiple logistic regressions and errors-in-variables regression models were employed to explore the associations between socioprovincial factors and mental disorders. Poisson regressions and errors-in-variables regression models were adopted to reveal the associations between socioprovincial factors and number of self-reported mental disorders.

Results: Descriptive statistics showed that mental health was poor in rural adolescents in China. Logistic regression showed that the odds of overall mental disorder and study anxiety were $189 \%(\mathrm{AOR}=2.89,95 \% \mathrm{Cl}: 2.76,3.02)$ and $92 \%(\mathrm{OR}=1.92,95 \% \mathrm{Cl}: 1.84,2.00)$ in Gansu more than those in Anhui, while the odds of personal anxiety, guilt, sensitivity, symptomatic psychosis, and phobia were $92 \%(A O R=0.08,95 \% \mathrm{Cl}: 0.08,0.09), 71 \%(\mathrm{AOR}=0.29,95 \% \mathrm{Cl}$ : $0.27,0.30), 88 \%(\mathrm{AOR}=0.12,95 \% \mathrm{Cl}: 0.11,0.13), 69 \%(\mathrm{AOR}=0.31,95 \% \mathrm{Cl}: 0.29,0.32)$, and $78 \%$ (AOR $=0.22,95 \% \mathrm{Cl}: 0.21$, 0.23 ) in Gansu less than those in Anhui. Moreover, Gansu (Poisson regression: IRR $=1.45$, 95\%Cl: 1.42-1.47; errors-invariables regression: Coefficient $=0.26,95 \% \mathrm{Cl}$ : 0.16, 0.36), Ningxia (Poisson regression: IRR $=1.63,95 \% \mathrm{Cl}$ : 1.60-1.67; errors-in-variables regression: Coefficient $=0.43,95 \% \mathrm{Cl}$ : 0.32, 0.53), Qinghai (Poisson regression: IRR $=1.65,95 \% \mathrm{Cl}$ : 1.60-1.69; errors-in-variables regression: Coefficient $=0.44,95 \% \mathrm{Cl}: 0.34,0.55)$, and Shaanxi (Poisson regression: IRR $=$ 1.28, 95\%Cl: 1.25-1.30; errors-in-variables regression: Coefficient $=0.11,95 \% \mathrm{Cl}: 0.00,0.21$ ) were significantly associated with the number of self-reported mental disorders.

(Continued on next page)
\end{abstract}

Correspondence: gming0604@163.com

'International Issues Center, Xuchang University, Xuchang, Road Bayi 88, Xuchang, Henan, China

${ }^{2}$ Family Issues Center, Xuchang University, Road Bayi 88, Xuchang, Henan,

China

Full list of author information is available at the end of the article

(c) The Author(s). 2021 Open Access This article is licensed under a Creative Commons Attribution 4.0 International License, which permits use, sharing, adaptation, distribution and reproduction in any medium or format, as long as you give appropriate credit to the original author(s) and the source, provide a link to the Creative Commons licence, and indicate if changes were made. The images or other third party material in this article are included in the article's Creative Commons licence, unless indicated otherwise in a credit line to the material. If material is not included in the article's Creative Commons licence and your intended use is not permitted by statutory regulation or exceeds the permitted use, you will need to obtain permission directly from the copyright holder. To view a copy of this licence, visit http://creativecommons.org/licenses/by/4.0/ The Creative Commons Public Domain Dedication waiver (http://creativecommons.org/publicdomain/zero/1.0/) applies to the data made available in this article, unless otherwise stated in a credit line to the data. 
(Continued from previous page)

Conclusion: Class and provincial disparities in self-reported mental disorders were reported among the students from Grade 4 to 8 in rural China. Mental health care supported by governments and schools could be an effective way to reduce the disparities in mental disorders among the adolescents.

Keywords: Socioprovincial factors, Self-reported mental disorders, Students from grade 4 to 8 , Number of selfreported mental disorders, Rural China

\section{Background}

There was a growing body of evidence suggesting that adolescents were susceptible to a variety of mental disorders among adolescents. Currently, mental disorders including anxiety disorders [1] and depressive symptoms [2] in adolescents had been attracted by academic circle. For example, an epidemiological study found a high incidence of comorbidity in children and adolescent psychiatry [3]. Another study indicated that depressive and anxiety symptoms often co-occurred [4]. Empirically, early adolescent anxiety disorders were related to lower self-esteem from early adolescence through young adulthood, with social phobia having the greatest impact [5].

Regarding socio-geographical factors, prior research reported that socioregional factors [6], social gradient in antisocial behavior [7], teacher perspectives [8], and income change [9] had associations with mental disorders among adolescents. Furthermore, a study reported race moderated the link between physical discipline and externalizing behavior problems for European American adolescents during Grades 6 and 8 [10]. Another large epidemiological study in Israel found an association between religion/ethnicity and internalizing and externalizing disorders in Muslim adolescents [11].

Multiple studies documented the associations between household wealth and mental health changes among adolescents worldwide. For instance, a study concluded that national wealth had associations with poor mental health at the aggregated level [12]. Regarding the association between wealth inequality and the risk of mental disability in the Chinese population, a study with nationally represented, population-based data from the second China National Sample Survey on Disability 2006 suggested that wealth was a significant predictor in the distribution of mental disability [13]. Furthermore, a study with data from 2060 young adults aged 18-27 in 20052011 from the Panel Study of Income Dynamics suggested family wealth affected mental health [14]. Likewise, a study with data (2001-2012) from the Household, Income and Labour Dynamics in Australia survey reported that low household wealth prior to disability acquisition in adulthood resulted in a greater deterioration in mental health than those with high wealth [15]. Additionally, a study with the case of three distinctive communities in Haiti found household agricultural wealth was significantly and strongly associated with both reductions in depression symptoms and anxiety symptoms [16].

However, there were a limited number of studies to report the associations between socioprovincial factors and self-reported mental disorders in rural China. Prior research on the associations of socioprovincial factors with mental health were seldom documented because the data employed did not include provincial variable. This study tried to fill into the gaps.

\section{Methods \\ Data source}

This study employed a publicly available survey data [17]. The dataset was aggregated from 10 different school-level surveys that conducted in rural areas of five provinces of from 2008 to 2015, which covered 54,498 students from Grade 4 to 8 across 65 counties in rural China. The dataset included information on student characteristics (gender, grade, and surveyed provinces) and family background (household asset) as well as mental health situations.

In order to measure psychological test of well-being, this data employed Mental Health Test (MHT) which was adapted by Professor Zhou Bucheng of East China Normal University from the General Anxiety Test developed by Kiyoshi Suzuki in Japan as an internationally standardized test for anxiety in children. MHT was a 100-item self-report inventory, in which consisted of 10item overall mental disorder (82, 84, 86, 88, 90, 92, 94, 96,98 , and 100), 15-item study anxiety $(1,2,3,4,5,6,7$, $8,9,10,11,12,13,14$, and 15), 10-item personal anxiety $(16,17,18,19,20,21,22,23,24$, and 25), 10-item loneliness $(26,27,28,29,30,31,32,33,34$, and 35), 10-item guilty (36, 37, 38, 39, 40, 41, 42, 43, 44, and 45), 10-item sensitivity $(46,47,48,49,50,51,52,53,54$, and 55), 15item symptomatic psychosis $(56,57,58,59,60,61,62$, $63,64,65,66,67,68,69$, and 70$), 10$-item phobia $(71$, $72,73,74,75,76,77,78,79$, and 80$)$, and 10-item impulsivity $(81,83,85,87,89,91,93,95,97$, and 99). The response options of each item were yes $(=1)$ and no $(=$ 0 ). Thus, the maximum subscale scores of overall mental disorder, study anxiety, personal anxiety, loneliness, guilty, sensitivity, symptomatic psychosis, phobia, and impulsivity were 10 points, 15 points, 10 points, 10 
points, 10 points, 10 points, 15 points, 10 points, and 10 points, respectively. Here, cut-off point of 7 was implemented in the subcategories of overall mental disorder, study anxiety, personal anxiety, loneliness, guilty, sensitivity, symptomatic psychosis, phobia, and impulsivity. Simultaneously, the eight subscales were divided by 7 and more points (yes $=1$ ) less than 7 points and (no = $0)$. The key variables' definition, scoring, and standardization were descripted in literature [18].

\section{Main variables}

Here, dependent variables referred to overall mental disorder, mental subscales (study anxiety, personal anxiety, loneliness, guilt, sensitivity, symptomatic psychosis, phobia, and impulsivity), and number of self-reported mental disorders. Additionally, overall mental disorder and mental subscales were reflected by the questions: "Percentage of kids who have $7+$ points in any category" and "Whether or not kids have 7+ points in study anxiety, personal anxiety, lonely, guilty, sensitive, symptomatic, phobic, or impulsive subcategory", respectively. Their response options were yes $(=1)$ and no $(=0)$. Number of self-reported mental disorders was calculated by arithmetic sum of response options of study anxiety, personal anxiety, loneliness, guilt, sensitivity, symptomatic psychosis, phobia, and impulsivity.

Independent variables included school grade $(4,5,7$, and 8 ), gender (female $=0$, male $=1$ ), surveyed provinces (Anhui, Gansu, Ningxia, Qinghai, and Shaanxi), and standardized index for household wealth. Standardized index for household wealth was constructed by polychoric principal components analysis with dichotomous household items, such as a microwave, refrigerator, fan, etc.

\section{Statistical analysis}

The associations between socioprovincial factors and self-reported mental disorders were mainly calculated by multiple logistic regressions. Due to response options with rare "yes" relative to "no" in loneliness and impulsivity, the associations between socioprovincial factors and mental disorders were calculated by complementary log-log regression (stata program: cloglog). The associations between socioprovincial factors and number of self-reported mental disorders were explored by Poisson regression with incidence-rate ratios (IRR).

Due to survey data, measurement errors could be considered in the errors-in-variables regression models when the subjective response variables were possibly measured with errors. Here, the assumed reliability of standardized index for household wealth was defined as 0.85 . Subsequently, errors-in-variables regressions on associations between socioprovincial factors and mental disorders were conducted.

\section{Results}

\section{Descriptive statistics}

Among the available 54,498 participants, males accounted for 52.04\%, and females accounted for $47.96 \%$. Regarding surveyed provinces, the majority of the sample was from Shaanxi (49.50\%), followed by Gansu (26.30\%), Ningxia (14.64\%), Qinghai (6.88\%), and Anhui (2.68\%). Regarding school grades, the majority of the sample were in Grade 4 (47.22\%), followed by Grade 5 (33.07\%), Grade 8 (10.09\%), and Grade 7 (9.63\%). Regarding mental morbidity, the number of self-reported mental disorders was distributed as $0(29.42 \%), 1$ (33.89\%), 2 (20.12\%), 3 (9.20\%), 4 (4.38\%), 5 (1.95\%), 6 (0.79\%), $7(0.22 \%)$, and $8(0.02 \%)$. In Table 1 , there were significant gender differences in the case of surveyed provinces, school grade, overall mental disorder, study anxiety, personal anxiety, loneliness, guilt, sensitivity, symptomatic psychosis, phobia, and impulsivity.

\section{Associations between socioprovincial factors and mental disorders}

Regarding gender, in Tables 2 and 3, significant adjusted odds ratios (AOR) and significant coefficients of male indicated male students were less susceptible to mental disorders than female students. Considering school grade, in Tables 2 and 3, significant adjusted odds ratios and significant coefficients indicated students in Grade 5 were less susceptible to mental disorders than students in Grade 4. In addition, students in Grades 7 and 8 were more susceptible to mental disorders than students in Grade 4.

Regarding surveyed provinces in Table 2, the odds of overall mental disorder and study anxiety were 189\% $(\mathrm{AOR}=2.89,95 \% \mathrm{CI}: 2.76,3.02)$ and $92 \%(\mathrm{OR}=1.92$, 95\%CI: 1.84, 2.00) in Gansu more than those in Anhui, respectively, while the odds of personal anxiety, guilt, sensitivity, symptomatic psychosis, and phobia were $92 \%$ $(\mathrm{AOR}=0.08, \quad 95 \% \mathrm{CI}: \quad 0.08, \quad 0.09), \quad 71 \% \quad(\mathrm{AOR}=0.29$, 95\%CI: $0.27,0.30), 88 \%(\mathrm{AOR}=0.12,95 \% \mathrm{CI}: 0.11,0.13)$, $69 \%(\mathrm{AOR}=0.31,95 \% \mathrm{CI}: 0.29,0.32)$, and $78 \%(\mathrm{AOR}=$ $0.22,95 \% \mathrm{CI}: 0.21,0.23$ ) in Gansu less than those in Anhui, respectively. Similarly, the odds of overall mental disorder and study anxiety were $255 \%(\mathrm{AOR}=3.55$, 95\%CI: $3.35,3.76$ ) and $115 \%$ (AOR $=2.15,95 \% \mathrm{CI}: 2.04$, 2.27) in Ningxia more than those in Anhui, respectively, while the odds of personal anxiety, guilt, sensitivity, symptomatic psychosis, and phobia were $90 \%(\mathrm{AOR}=$ $0.10,95 \%$ CI: $0.09,0.11), 70 \%$ (AOR $=0.30,95 \% \mathrm{CI}: 0.29$, $0.32), 86 \%(\mathrm{AOR}=0.14,95 \% \mathrm{CI}: 0.13,0.15), 57 \%(\mathrm{AOR}=$ $0.43,95 \% \mathrm{CI}: 0.41,0.46)$, and $70 \%(\mathrm{AOR}=0.30,95 \% \mathrm{CI}$ : $0.28,0.32)$ in Ningxia less than those in Anhui, respectively. Likewise, the odds of overall mental disorder and study anxiety were $345 \%(\mathrm{AOR}=4.45,95 \% \mathrm{CI}: 4.09,4.84)$ and $143 \%(\mathrm{AOR}=2.43,95 \% \mathrm{CI}: 2.26,2.62)$ in Qinghai 
Table 1 Sample characteristics by gender

\begin{tabular}{|c|c|c|c|c|c|c|}
\hline & \multicolumn{2}{|c|}{ Female } & \multicolumn{2}{|l|}{ Male } & \multirow{2}{*}{$\begin{array}{l}\text { Chi- } \\
\text { square }\end{array}$} & \multirow[t]{2}{*}{$P$ value } \\
\hline & $\mathrm{N}$ & $\%$ & $\mathrm{~N}$ & $\%$ & & \\
\hline Surveyed provinces $(N=54,498)$ & & & & & 35.5147 & $0.000^{* * *}$ \\
\hline Anhui & 715 & 1.31 & 746 & 1.37 & & \\
\hline Gansu & 7098 & 13.02 & 7234 & 13.27 & & \\
\hline Ningxia & 3924 & 7.20 & 4057 & 7.44 & & \\
\hline Qinghai & 1793 & 3.29 & 1956 & 3.59 & & \\
\hline Shaanxi & 12,607 & 23.13 & 14,368 & 26.36 & & \\
\hline School Grade $(N=54,498)$ & & & & & 14.4172 & $0.002^{* * *}$ \\
\hline 4 & 12,314 & 22.60 & 13,420 & 24.62 & & \\
\hline 5 & 8811 & 16.17 & 9209 & 16.90 & & \\
\hline 7 & 2428 & 4.46 & 2819 & 5.17 & & \\
\hline 8 & 2584 & 4.74 & 2913 & 5.35 & & \\
\hline Standardized index for household wealth (median, Interquartile Range) $(N=48,956)$ & \multicolumn{2}{|c|}{$\begin{array}{l}-.2809,(-.6637 \\
.8124)\end{array}$} & \multicolumn{2}{|c|}{$\begin{array}{l}-.2611,(-.6459 \\
.8700)\end{array}$} & & \\
\hline Mental disorder $(N=54,498)$ & & & & & 268.4976 & $0.000^{* * *}$ \\
\hline No & 6819 & 12.51 & 9215 & 16.91 & & \\
\hline Yes & 19,318 & 35.45 & 19,146 & 35.13 & & \\
\hline Study anxiety $(N=54,498)$ & & & & & 205.0397 & $0.000^{* * *}$ \\
\hline No & 9212 & 16.90 & 11,689 & 21.45 & & \\
\hline Yes & 16,925 & 31.06 & 16,672 & 30.59 & & \\
\hline Personal anxiety $(N=54,498)$ & & & & & 60.9713 & $0.000^{* * *}$ \\
\hline No & 24,169 & 44.35 & 26,699 & 48.99 & & \\
\hline Yes & 1968 & 3.61 & 1662 & 3.05 & & \\
\hline Loneliness ( $N=54,498)$ & & & & & 1.7715 & 0.183 \\
\hline No & 25,640 & 47.05 & 27,865 & 51.13 & & \\
\hline Yes & 497 & 0.91 & 496 & 0.91 & & \\
\hline Guilt $(N=54,498)$ & & & & & 342.9068 & $0.000^{* * *}$ \\
\hline No & 20,522 & 37.66 & 24,009 & 44.05 & & \\
\hline Yes & 5615 & 10.30 & 4352 & 7.99 & & \\
\hline Sensitivity $(N=54,498)$ & & & & & 3.9022 & $0.048^{* *}$ \\
\hline No & 22,928 & 42.07 & 25,035 & 45.94 & & \\
\hline Yes & 3209 & 5.89 & 3326 & 6.10 & & \\
\hline Symptomatic psychosis $(N=54,498)$ & & & & & 14.6444 & $0.000^{* * *}$ \\
\hline No & 20,441 & 37.51 & 22,560 & 41.40 & & \\
\hline Yes & 5696 & 10.45 & 5801 & 10.64 & & \\
\hline Phobia $(N=54,498)$ & & & & & $1.4 \mathrm{e}+03$ & $0.000^{* * *}$ \\
\hline No & 21,679 & 39.78 & 26,488 & 48.60 & & \\
\hline Yes & 4458 & 8.18 & 1873 & 3.44 & & \\
\hline Impulsivity ( $N=54,498)$ & & & & & 2.3446 & 0.126 \\
\hline No & 25,545 & 46.87 & 27,662 & 50.76 & & \\
\hline Yes & 592 & 1.09 & 699 & 1.28 & & \\
\hline
\end{tabular}

Note: ${ }^{*} p<0.1,{ }^{* *} p<0.05,{ }^{* * *} p<0.01$ 
Table 2 Logistic and linear regression on associations between socioprovincial factors and mental disorders. AOR (95\%Cl), Coefficient $(95 \% \mathrm{Cl})$

\begin{tabular}{|c|c|c|c|c|c|c|c|c|c|}
\hline & OMD & $\begin{array}{l}\text { Study } \\
\text { anxiety }\end{array}$ & $\begin{array}{l}\text { Personal } \\
\text { anxiety }\end{array}$ & Loneliness & Guilt & Sensitivity & $\begin{array}{l}\text { Symptomatic } \\
\text { psychosis }\end{array}$ & Phobia & Impulsivity \\
\hline \multicolumn{10}{|l|}{ Gender } \\
\hline Female & Reference & Reference & Reference & Reference & Reference & Reference & Reference & Reference & Reference \\
\hline Male & $\begin{array}{l}0.75^{* * *} \\
(0.72,0.78)\end{array}$ & $\begin{array}{l}0.77^{* * *} \\
(0.75,0.80)\end{array}$ & $\begin{array}{l}0.71^{* * *} \\
(0.67,0.77)\end{array}$ & $\begin{array}{l}-0.09(-0.22 \\
0.04)\end{array}$ & $\begin{array}{l}0.62^{* * *}(0.60, \\
0.65)\end{array}$ & $\begin{array}{l}0.86^{* * *}(0.81 \\
0.91)\end{array}$ & $0.87^{* * *}(0.84,0.91)$ & $\begin{array}{l}0.32^{* * *}(0.30, \\
0.34)\end{array}$ & $\begin{array}{l}0.05(-0.06 \\
0.17)\end{array}$ \\
\hline \multicolumn{10}{|l|}{ Grade } \\
\hline 4 & Reference & Reference & Reference & Reference & Reference & Reference & Reference & Reference & Reference \\
\hline 5 & $\begin{array}{l}0.99(0.95, \\
1.04)\end{array}$ & $\begin{array}{l}1.01(0.97 \\
1.05)\end{array}$ & $\begin{array}{l}0.90^{* *}(0.83, \\
0.98)\end{array}$ & $\begin{array}{l}-0.23^{* * *}(- \\
0.39,-0.08)\end{array}$ & $\begin{array}{l}0.93^{* * *}(0.88, \\
0.97)\end{array}$ & $\begin{array}{l}0.87^{* * *}(0.81 \\
0.92)\end{array}$ & $0.78^{* * *}(0.74,0.81)$ & $\begin{array}{l}0.81^{* * *}(0.76 \\
0.86)\end{array}$ & $\begin{array}{l}-0.03(-0.17 \\
0.11)\end{array}$ \\
\hline 7 & $\begin{array}{l}1.54^{* * *} \\
(1.43,1.67)\end{array}$ & $\begin{array}{l}1.51^{* * *} \\
(1.41,1.63)\end{array}$ & $\begin{array}{l}1.37^{* * *} \\
(1.19,1.58)\end{array}$ & $\begin{array}{l}0.39^{* * * *}(0.13, \\
0.64)\end{array}$ & $\begin{array}{l}1.13^{* * *(1.03,} \\
1.24)\end{array}$ & $\begin{array}{l}1.26^{* * *(1.13} \\
1.39)\end{array}$ & $1.26^{* * *}(1.15,1.38)$ & $\begin{array}{l}0.91 \text { (0.81, } \\
1.03)\end{array}$ & $\begin{array}{l}0.74^{* * *}(0.53, \\
0.96)\end{array}$ \\
\hline 8 & $\begin{array}{l}1.74^{* * *} \\
(1.61,1.88)\end{array}$ & $\begin{array}{l}1.65^{* * *} \\
(1.54,1.78)\end{array}$ & $\begin{array}{l}1.57^{* * *} \\
(1.38,1.80)\end{array}$ & $\begin{array}{l}0.60^{* * *}(0.36 \\
0.84)\end{array}$ & $\begin{array}{l}1.15^{* * *(1.05} \\
1.26)\end{array}$ & 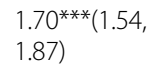 & $1.42^{* * *}(1.31,1.55)$ & $\begin{array}{l}0.93(0.83 \\
1.04)\end{array}$ & $\begin{array}{l}0.87^{* * *}(0.66, \\
1.07)\end{array}$ \\
\hline \multicolumn{10}{|c|}{ Surveyed provinces } \\
\hline Anhui & Reference & Reference & Reference & Reference & Reference & Reference & Reference & Reference & Reference \\
\hline Gansu & $\begin{array}{l}2.89^{* * *} \\
(2.76,3.02)\end{array}$ & $\begin{array}{l}1.92^{* * *} \\
(1.84,2.00)\end{array}$ & $\begin{array}{l}0.08^{* * *} \\
(0.08,0.09)\end{array}$ & $\begin{array}{l}0.71^{*}(-0.02, \\
1.44)\end{array}$ & $\begin{array}{l}0.29^{* * *}(0.27 \\
0.30)\end{array}$ & $\begin{array}{l}0.12^{* * *}(0.11 \\
0.13)\end{array}$ & $0.31^{* * *}(0.29,0.32)$ & $\begin{array}{l}0.22^{* * *}(0.21, \\
0.23)\end{array}$ & $\begin{array}{l}0.37^{* * *}(-0.21 \\
0.94)\end{array}$ \\
\hline Ningxia & $\begin{array}{l}3.55^{* * *} \\
(3.35,3.76)\end{array}$ & $\begin{array}{l}2.15^{* * *} \\
(2.04,2.27)\end{array}$ & $\begin{array}{l}0.10^{* * *} \\
(0.09,0.11)\end{array}$ & $\begin{array}{l}0.80^{* *}(0.06 \\
1.54)\end{array}$ & $\begin{array}{l}0.30^{* * *}(0.29, \\
0.32)\end{array}$ & $\begin{array}{l}0.14^{* * *}(0.13 \\
0.15)\end{array}$ & $0.43^{* * *}(0.41,0.46)$ & $\begin{array}{l}0.30^{* * *}(0.28, \\
0.32)\end{array}$ & $0.75(0.18,1.33)$ \\
\hline Qinghai & $\begin{array}{l}4.45^{* * *} \\
(4.09,4.84)\end{array}$ & $\begin{array}{l}2.43^{* * *} \\
(2.26,2.62)\end{array}$ & $\begin{array}{l}0.09^{* * *} \\
(0.08,0.10)\end{array}$ & $\begin{array}{l}1.16^{* * *}(0.41 \\
1.90)\end{array}$ & $\begin{array}{l}0.26^{* * *}(0.23, \\
0.28)\end{array}$ & $\begin{array}{l}0.12^{* * *}(0.11 \\
0.13)\end{array}$ & $0.54^{* * *}(0.50,0.59)$ & $\begin{array}{l}0.26^{* * *}(0.23 \\
0.29)\end{array}$ & $\begin{array}{l}1.11^{* * *}(0.53, \\
1.69)\end{array}$ \\
\hline Shaanxi & $\begin{array}{l}1.92^{* * *} \\
(1.83,2.00)\end{array}$ & $\begin{array}{l}1.31^{* * *} \\
(1.26,1.37)\end{array}$ & $\begin{array}{l}0.07^{* * *} \\
(0.07,0.08)\end{array}$ & $0.47(-0.27,1.20)$ & $\begin{array}{l}0.26^{* * *}(0.25 \\
0.27)\end{array}$ & $\begin{array}{l}0.15^{* * *}(0.14 \\
0.16)\end{array}$ & $0.22^{* * *}(0.21,0.23)$ & $\begin{array}{l}0.20^{* * *}(0.19 \\
0.22)\end{array}$ & $0.33(-0.24,0.91)$ \\
\hline $\mathrm{SIHW}$ & $\begin{array}{l}1.00^{* * *} \\
(1.00,1.00)\end{array}$ & $\begin{array}{l}1.00^{* *}(1.00, \\
1.00)\end{array}$ & $\begin{array}{l}1.00^{* * *} \\
(1.00,1.00)\end{array}$ & $0.00(-0.00,0.00)$ & $\begin{array}{l}1.00^{* * *}(1.00, \\
1.00)\end{array}$ & 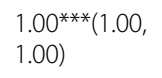 & $1.00^{* * *}(1.00,1.00)$ & $\begin{array}{l}1.00^{* * *(1.00}, \\
1.00)\end{array}$ & $\begin{array}{l}0.00^{* *}(0.00, \\
0.00)\end{array}$ \\
\hline Constant & & & & $\begin{array}{l}-4.62^{* * *}(-5.35,- \\
3.88)\end{array}$ & & & & & $\begin{array}{l}-4.43^{* * *}(-5.00, \\
-3.85)\end{array}$ \\
\hline $\mathrm{N}$ & 48,956 & 48,956 & 48,956 & 48,956 & 48,956 & 48,956 & 48,956 & 48,956 & 48,956 \\
\hline
\end{tabular}

Note: ${ }^{*} p<0.1,{ }^{* *} p<0.05,{ }^{* * *} p<0.01$. SIHW Standardized index for household wealth. OMD Overall mental disorder

more than those in Anhui, respectively, while the odds of personal anxiety, guilt, sensitivity, symptomatic psychosis, and phobia were 91\% (AOR $=0.09,95 \% \mathrm{CI}: 0.08$, $0.10), 74 \%(\mathrm{AOR}=0.26,95 \% \mathrm{CI}: 0.23,0.28), 88 \%(\mathrm{AOR}=$ 0.12, 95\% CI: 0.11, 0.13), 46\% (AOR $=0.54,95 \% \mathrm{CI}: 0.50$, 0.59 ), and $74 \%$ (AOR $=0.26,95 \% \mathrm{CI}: 0.23,0.29)$ in Qinghai less than those in Anhui, respectively. Additionally, the odds of overall mental disorder and study anxiety were $92 \%(\mathrm{AOR}=1.92,95 \% \mathrm{CI}: 1.83,2.00)$ and $31 \%$ $(\mathrm{AOR}=1.31,95 \% \mathrm{CI}: 1.26,1.37)$ in Shaanxi more than those in Anhui, respectively, while the odds of personal anxiety, guilt, sensitivity, symptomatic psychosis, and phobia were $93 \%$ (AOR $=0.07,95 \% \mathrm{CI}: 0.07,0.08), 74 \%$ $(\mathrm{AOR}=0.26, \quad 95 \% \mathrm{CI}: \quad 0.25, \quad 0.27), \quad 85 \% \quad(\mathrm{AOR}=0.15$, 95\%CI: $0.14,0.16), 78 \%(\mathrm{AOR}=0.22,95 \% \mathrm{CI}: 0.21,0.23$ ), and $80 \%(\mathrm{AOR}=0.20,95 \% \mathrm{CI}: 0.19,0.22)$ in Shaanxi less than those in Anhui, respectively. Significantly positive and negative coefficients in Table 3 basically reflected the similar associations.

Simultaneously, in Tables 2 and 3, significant adjusted odds ratios and significant coefficients indicated standardized index for household wealth had no associations with mental disorders.

\section{Associations between socioprovincial factors and number of self-reported mental disorders}

In Table 4, male was significantly associated with the number of self-reported mental disorders (Poisson regression: $\quad \mathrm{IRR}=0.82, \quad 95 \% \mathrm{CI}: \quad 0.81-0.84 ; \quad$ errors-invariables regression: Coefficient $=-0.27,95 \% \mathrm{CI}:-0.29$, - 0.24). Regarding school grade, Grades 7 (Poisson regression: IRR $=1.22,95 \% \mathrm{CI}$ : $1.18-1.26$; errors-invariables regression: Coefficient $=0.25,95 \% \mathrm{CI}: 0.20$, 0.30 ) and 8 (Poisson regression: IRR $=1.31,95 \% \mathrm{CI}$ : $1.27-$ 1.35; errors-in-variables regression: Coefficient $=0.36$, $95 \%$ CI: $0.31,0.40)$ were significantly associated with the number of self-reported mental disorders. Regarding surveyed provinces, Gansu (Poisson regression: IRR = 1.45, 95\%CI: 1.42-1.47; errors-in-variables regression: Coefficient $=0.26,95 \%$ CI: 0.16, 0.36), Ningxia (Poisson regression: $\mathrm{IRR}=1.63,95 \% \mathrm{CI}$ : $1.60-1.67$; errors-invariables regression: Coefficient $=0.43,95 \% \mathrm{CI}$ : 0.32 , 
Table 3 Errors-in-variables regression on associations between socioprovincial factors and mental disorders, Coefficient (95\%Cl)

\begin{tabular}{|c|c|c|c|c|c|c|c|c|c|}
\hline & OMD & Study anxiety & $\begin{array}{l}\text { Personal } \\
\text { anxiety }\end{array}$ & Loneliness & Guilt & Sensitivity & $\begin{array}{l}\text { Symptomatic } \\
\text { psychosis }\end{array}$ & Phobia & Impulsivity \\
\hline \multicolumn{10}{|l|}{ Gender } \\
\hline Female & Reference & Reference & Reference & Reference & Reference & Reference & Reference & Reference & Reference \\
\hline Male & $\begin{array}{l}-0.06^{* * *}(-0.07 \\
-0.06)\end{array}$ & $\begin{array}{l}-0.06^{* * *}(- \\
0.07,-0.05)\end{array}$ & $\begin{array}{l}-0.02^{* * *}(- \\
0.02,-0.01)\end{array}$ & $\begin{array}{l}-0.00(-0.00 \\
0.00)\end{array}$ & $\begin{array}{l}-0.06^{* * *}(-0.07 \\
-0.06)\end{array}$ & $\begin{array}{l}-0.01^{* *}(-0.01 \\
-0.00)\end{array}$ & $\begin{array}{l}-0.01^{* * *}(-0.02 \\
-0.01)\end{array}$ & $\begin{array}{l}-0.11^{* * *}(-0.11 \\
-0.10)\end{array}$ & $\begin{array}{l}0.00(-0.00 \\
0.00)\end{array}$ \\
\hline \multicolumn{10}{|l|}{ Grade } \\
\hline 4 & Reference & Reference & Reference & Reference & Reference & Reference & Reference & Reference & Reference \\
\hline 5 & $\begin{array}{l}-0.01 * * *(- \\
0.02,-0.01)\end{array}$ & $\begin{array}{l}0.00(-0.01 \\
0.01)\end{array}$ & $\begin{array}{l}0.00(-0.00 \\
0.01)\end{array}$ & $\begin{array}{l}-0.00^{* * *}(- \\
0.01,-0.00)\end{array}$ & $\begin{array}{l}0.01 *(-0.00, \\
0.02)\end{array}$ & $\begin{array}{l}0.01 * * * * 0.01 \\
0.02)\end{array}$ & $\begin{array}{l}-0.02 * * * \\
-0.01)\end{array}$ & $\begin{array}{l}-0.00(-0.01 \\
0.00)\end{array}$ & $\begin{array}{l}-0.00(- \\
0.00,0.00)\end{array}$ \\
\hline 7 & $\begin{array}{l}0.09^{* * * *}(0.07 \\
0.11)\end{array}$ & $\begin{array}{l}0.10^{* * * *}(0.08, \\
0.12)\end{array}$ & $\begin{array}{l}0.02 * * * \\
0.03)\end{array}$ & $\begin{array}{l}0.01 * * *(0.00, \\
0.01)\end{array}$ & $\begin{array}{l}0.03^{* * *}(0.01 \\
0.04)\end{array}$ & $\begin{array}{l}0.04^{* * *}(0.03, \\
0.05)\end{array}$ & $\begin{array}{l}0.04^{* * *}(0.03, \\
0.06)\end{array}$ & $\begin{array}{l}-0.00(-0.01 \\
0.01)\end{array}$ & $\begin{array}{l}0.02^{* * *} \\
(0.01,0.02)\end{array}$ \\
\hline 8 & $\begin{array}{l}0.11^{* * *}(0.10, \\
0.13)\end{array}$ & $\begin{array}{l}0.12^{* * *}(0.10, \\
0.14)\end{array}$ & $\begin{array}{l}0.03^{* * * *}(0.02, \\
0.04)\end{array}$ & $\begin{array}{l}0.01^{* * *}(0.01 \\
0.02)\end{array}$ & $\begin{array}{l}0.03^{* * *}(0.01 \\
0.04)\end{array}$ & $\begin{array}{l}0.08^{* * * *}(0.07, \\
0.09)\end{array}$ & $\begin{array}{l}0.06^{* * *}(0.05, \\
0.08)\end{array}$ & $\begin{array}{l}-0.00(-0.01 \\
0.01)\end{array}$ & $\begin{array}{l}0.02^{* * *} \\
(0.02,0.03)\end{array}$ \\
\hline \multicolumn{10}{|c|}{ Surveyed provinces } \\
\hline Anhui & Reference & Reference & Reference & Reference & Reference & Reference & Reference & Reference & Reference \\
\hline Gansu & $\begin{array}{l}0.10^{* * *}(0.06, \\
0.13)\end{array}$ & $\begin{array}{l}0.14^{* * *}(0.10 \\
0.18)\end{array}$ & $\begin{array}{l}0.02^{*}(-0.00, \\
0.04)\end{array}$ & $\begin{array}{l}0.01 *(-0.00, \\
0.02)\end{array}$ & $\begin{array}{l}-0.01(-0.04 \\
0.02)\end{array}$ & $\begin{array}{l}0.01(-0.02 \\
0.03)\end{array}$ & $\begin{array}{l}0.07^{* * *}(0.04, \\
0.11)\end{array}$ & $\begin{array}{l}0.02(-0.00 \\
0.04)\end{array}$ & $\begin{array}{l}0.01(-0.00, \\
0.02)\end{array}$ \\
\hline Ningxia & $\begin{array}{l}0.14^{* * *}(0.10, \\
0.17)\end{array}$ & $\begin{array}{l}0.17^{* * *}(0.13, \\
0.21)\end{array}$ & $\begin{array}{l}0.03^{* * * *}(0.01 \\
0.05)\end{array}$ & $\begin{array}{l}0.01 *(-0.00, \\
0.02)\end{array}$ & $\begin{array}{l}-0.01(-0.04 \\
0.03)\end{array}$ & $\begin{array}{l}0.02(-0.01 \\
0.05)\end{array}$ & $\begin{array}{l}0.14^{* * *}(0.10, \\
0.17)\end{array}$ & $\begin{array}{l}0.05^{* * * *}(0.03, \\
0.08)\end{array}$ & $\begin{array}{l}0.02^{* * *} \\
(0.00,0.03)\end{array}$ \\
\hline Qinghai & $\begin{array}{l}0.18^{* * *}(0.14 \\
0.22)\end{array}$ & $\begin{array}{l}0.19^{* * *}(0.15 \\
0.23)\end{array}$ & $\begin{array}{l}0.02^{* *}(0.00, \\
0.04)\end{array}$ & $0.02(0.01,0.03)$ & $\begin{array}{l}-0.03 *(-0.06, \\
0.00)\end{array}$ & $\begin{array}{l}0.00(-0.03 \\
0.03)\end{array}$ & $\begin{array}{l}0.18^{* * * *}(0.14 \\
0.21)\end{array}$ & $\begin{array}{l}0.03^{* *}(0.01 \\
0.06)\end{array}$ & $\begin{array}{l}0.03^{* * *} \\
(0.01,0.04)\end{array}$ \\
\hline Shaanxi & $0.01(-0.03,0.04)$ & $\begin{array}{l}0.05^{* *}(0.01 \\
0.09)\end{array}$ & $0.01(-0.01,0.03)$ & $0.01(-0.01,0.02)$ & $\begin{array}{l}-0.03^{*}(-0.06 \\
0.00)\end{array}$ & $\begin{array}{l}0.02^{*}(-0.00 \\
0.05)\end{array}$ & $0.03(-0.01,0.06)$ & $\begin{array}{l}0.01(-0.01 \\
0.04)\end{array}$ & $\begin{array}{l}0.01(-0.00 \\
0.02)\end{array}$ \\
\hline SIHW & $0.00(-0.00,0.00)$ & $0.00(-0.00,0.00)$ & $\begin{array}{l}-0.00(-0.00 \\
0.00)\end{array}$ & $\begin{array}{l}0.00(-0.00, \\
0.00)\end{array}$ & $\begin{array}{l}-0.00(-0.00 \\
0.00)\end{array}$ & $\begin{array}{l}0.00(-0.00 \\
0.00)\end{array}$ & $0.00(-0.00,0.00)$ & $\begin{array}{l}0.00(-0.00 \\
0.00)\end{array}$ & $\begin{array}{l}0.00^{* *}(0.00, \\
0.00)\end{array}$ \\
\hline Constant & $\begin{array}{l}0.65^{* * *}(0.62, \\
0.69)\end{array}$ & $\begin{array}{l}0.52^{* * * *}(0.48, \\
0.56)\end{array}$ & $\begin{array}{l}0.05^{* * * *}(0.03 \\
0.07)\end{array}$ & $\begin{array}{l}0.01^{* *}(0.00, \\
0.02)\end{array}$ & $\begin{array}{l}0.22^{* * *}(0.19, \\
0.25)\end{array}$ & $\begin{array}{l}0.09^{* * * *}(0.06, \\
0.11)\end{array}$ & $\begin{array}{l}0.15^{* * *}(0.11 \\
0.18)\end{array}$ & $\begin{array}{l}0.15^{* * *}(0.12 \\
0.17)\end{array}$ & $\begin{array}{l}0.01(-0.00 \\
0.02)\end{array}$ \\
\hline $\begin{array}{l}\text { R- } \\
\text { squared }\end{array}$ & 0.0200 & 0.0158 & 0.0032 & 0.0014 & 0.0075 & 0.0075 & 0.0137 & 0.0297 & 0.0034 \\
\hline $\mathrm{N}$ & 48,956 & 48,956 & 48,956 & 48,956 & 48,956 & 48,956 & 48,956 & 48,956 & 48,956 \\
\hline
\end{tabular}

Note: ${ }^{*} p<0.1,{ }^{* *} p<0.05,{ }^{* * *} p<0.01$. SIHW Standardized index for household wealth. OMD Overall mental disorder

0.53), Qinghai (Poisson regression: IRR $=1.65,95 \% \mathrm{CI}$ : 1.60-1.69; errors-in-variables regression: Coefficient $=$ 0.44, 95\%CI: 0.34, 0.55), and Shaanxi (Poisson regression: $\mathrm{IRR}=1.28,95 \% \mathrm{CI}: 1.25-1.30$; errors-in-variables regression: Coefficient $=0.11,95 \% \mathrm{CI}: 0.00,0.21$ ) were significantly associated with the number of self-reported mental disorders. Also, standardized index for household wealth was significantly associated with the number of self-reported mental disorders (Poisson regression: IRR $=1.00,95 \%$ CI: $1.00-1.00$ ).

\section{Discussion}

Notably, high prevalence of self-reported mental disorders was reported among the sampled students. The associations between socioprovincial factors and selfreported mental disorders and the number of selfreported mental disorders were confirmed. Besides gender disparities in mental disorders, there existed class and provincial disparities in mental disorders among the sample.

Regarding gender, this study was consistent with a series of early studies. For example, a Jordan adolescents study reported significant associations of age and gender with high prevalence of mental disorders [19]. A study with data from China National Sample Surveys on Disability in 1987 and 2006 indicated that gender of children was consistently associated with psychiatric disability among children [20]. The finding in this study was also in line with a study in South Korea which reported adolescent depression experience was highly associated with gender mix in the school [21]. In this study, there might be gender disparities between mental disorders.

Regarding schooling, this study was in line with a series of early studies. For example, a study demonstrated important mental health issues with a high incidence of comorbidities in poverty-stricken areas and left-behind children and adolescents in 40 primary and middle schools [22]. Globally, a substantial study also documented high mental disorders prevalence among adolescent students in Brazilian [23], left behind adolescents in Anhui province [24], adolescent age groups in Saudi Arabia [25], Australian child and adolescents [26], Hungarian children [27], and Lithuanian youth [28]. 
Table 4 Associations between socioeconomic factors and number of mental disorders. IRR (95\%Cl), Coefficient (95\%Cl)

\begin{tabular}{|c|c|c|c|c|}
\hline & \multicolumn{2}{|c|}{ Poisson regression } & \multicolumn{2}{|c|}{ Errors-in-variables regression } \\
\hline & IRR & $95 \% \mathrm{Cl}$ & Coefficient & $95 \% \mathrm{Cl}$ \\
\hline \multicolumn{5}{|l|}{ Gender } \\
\hline Female & Reference & & Reference & \\
\hline Male & $0.82^{* * *}$ & $0.81,0.84$ & $-0.27^{* * *}$ & $-0.29,-0.24$ \\
\hline \multicolumn{5}{|l|}{ School Grade } \\
\hline 4 & Reference & & Reference & \\
\hline 5 & 1.01 & $0.99,1.03$ & -0.00 & $-0.03,0.02$ \\
\hline 7 & $1.22^{* * *}$ & $1.18,1.26$ & $0.25^{* * *}$ & $0.20,0.30$ \\
\hline 8 & $1.31^{* * *}$ & $1.27,1.35$ & $0.36^{* * *}$ & $0.31,0.40$ \\
\hline \multicolumn{5}{|l|}{ Surveyed provinces } \\
\hline Anhui & Reference & & Reference & \\
\hline Gansu & $1.45^{* * *}$ & $1.42,1.47$ & $0.26^{* * *}$ & $0.16,0.36$ \\
\hline Ningxia & $1.63^{* * *}$ & $1.60,1.67$ & $0.43^{* * *}$ & $0.32,0.53$ \\
\hline Qinghai & $1.65^{* * *}$ & $1.60,1.69$ & $0.44^{* * *}$ & $0.34,0.55$ \\
\hline Shaanxi & $1.28^{* * *}$ & $1.25,1.30$ & $0.11^{* *}$ & $0.00,0.21$ \\
\hline Standardized index for household wealth & $1.00^{* * *}$ & $1.00,1.00$ & 0.00 & $-0.00,0.00$ \\
\hline Constant & & & $1.20^{* * *}$ & $1.10,1.30$ \\
\hline Log likelihood & $-75,234.409$ & & & \\
\hline R-squared & & & 0.0212 & \\
\hline $\mathrm{N}$ & 48,956 & & 48,956 & \\
\hline
\end{tabular}

Note: ${ }^{*} p<0.1,{ }^{* *} p<0.05,{ }^{* * *} p<0.01$. SIHW Standardized index for household wealth

Additionally, an available research on the prevalence of child and adolescent psychiatric disorders in India was reviewed, synthesized, and evaluated [29]. The novel finding in this study was there existed class disparities in mental disorders among the students. Meanwhile, the students in higher class were more likely to be susceptible to mental disorders than those in lower class in junior school. The students in elementary school were less likely to be susceptible to mental disorders than those in junior school.

IRR of standardized index for household wealth was equal to 1 , which indicated that household wealth was not the main determinant of the number of mental disorders. This finding could be verified by a current research which reported family functioning had associations with adolescent health and emotional well-being [30]. Furthermore, the research outcome was not in congruent with the published associations between wealth inequality and positive emotion [31, 32].

To the best of the knowledge of the author, this was the first study to report provincial disparities of mental disorders in rural China. Some early studies documented mental health of school students in Hong Kong, Shanghai, and Beijing [33], Shanghai [34-36], and Sichuan [22]. Likewise, mental health in rural China also was reported in northwestern China [37] and western China [38]. Compared with these studies, the novel finding in this study highlighted provincial disparities of mental disorders in rural China.

\section{Policy implications}

This study highlighted the importance of socioprovincial factors in mental health. Adolescent mental disorders were confirmed to represent a risk marker for a number of later adverse outcomes [39]. Socioeconomic disadvantage and psychological deficits were found to contribute to criminal offending independently and with roughly equal magnitude [40]. Thus, adolescent psychopathology and support need be provided to minimize adverse outcomes. Regarding the provincial differences among ORs and IRRs, to my opinion, governments and schools should concern the differences of mental disorders between province-level units in China. Obviously, governments and schools were responsible for provincial imbalance of mental health service in rural China.

Understanding the associations between socioprovincial factors and self-reported mental disorders could help identify high-risk adolescents and take steps to minimize their disparities in mental disorders. Some similar studies recommended school mental health resources [41], developing personalized approaches to clinical assessment [42], adaptive interventions [43], and increasing health workforce [44] should be adopted to prevent and reduce the prevalence of mental disorders 
among the students. On the basis of the results in this study, optional prevention measures against mental orders need to smooth the difference of funds, human resource, and facilities among schools and provinces. Mental health care policies aimed at a redistribution of health resources at the provincial level also could decrease health service utilization inequalities in the adolescent mental well-beings.

\section{Limitations}

Some limitations in this study should be highlighted. First, this study employed a second-handed survey data which did not provided specific items of MHT. Thus, reliability (split-half/Cronbach's alpha, test-retest reliability, alternate form reliability, inter-rater reliability) and validity (content validity, construct validity, convergent or concurrent validity, discriminant validity, criterion (or predictive) validity) could not be calculated. Second, only five provinces were analyzed in this study. The research outcomes could be limitedly generalized to the whole country. Finally, several key definitions were ambiguous. Taking household wealth as an example, whether the wealth was parental or students' was not defined. Meanwhile, a study with data from the UK Millennium Cohort Study indicated children's mental health was not influenced by parental housing wealth but family permanent income and socioeconomic characteristics [45]. In addition, some key variables including socioeconomic inequalities [46], socioeconomic status [47], length of residence [48], unhealthy behaviours [49], and physiological engagement in social contexts [50], which were confirmed to be associated with adolescent mental disorders, were not included. Thus, mediating and moderating analyses could not be conducted. Accordingly, further research needs more variables to gain a better understanding of the disparities in student mental health.

\section{Conclusion}

In conclusion, this study reflected the associations between socioprovincial factors and self-reported mental disorders among students from Grade 4 to 8 in rural China. Especially, this study reported disparities in gender, class, and province regarding mental disorders. Regarding the situation reflected by this study, public policy intervention should be highlighted and redesigned to solve the mental disorders of the adolescents in rural areas of China.

\section{Abbreviations}

MHT: Mental Health Test; IRR: Incidence-rate ratios; AOR: Adjusted odds ratio; Cl: Confidence interval; SIHW: Standardized index for household wealth; OMD: Overall mental disorder

\section{Acknowledgements}

The author of this paper would like to acknowledge the very helpful comments of two reviewers on the original submission.

\section{Author's contributions}

MG designed the study, performed the statistical analysis, and completed the original version. The author(s) read and approved the final manuscript.

\section{Authors' information}

Ming Guan is head of the International Issues Center and Family Issues Center at Xuchang University. He is interested in health care service, health change, and quality of life of migrants and elders in modern China.

\section{Funding}

This project was funded by the Multi-dimensional Evaluation of Health Service System of Floating Population in Henan Province (in Chinese: 河南省流 动人口健康服务体系多维度评价研究; Project number: 2020BSH014) from 2020 Planning of Philosophy and Social Sciences in Henan Province (in Chinese: 2020年河南省哲学社会科学规划年度项目) and Research on the improvement of population mobility-driven health service system (in Chinese:

人口流动驱动的卫生服务体系改进研究; Project number: 2020ZD019) from 2020 Key Research Projects of Xuchang University (in Chinese: 2020年许昌学 院重点研究项目)

This project was also funded by the Construction Study and Practice of Ideological and Political Teaching in the Course of China Geography (in Chinese:《中国地理》课程思政建设研究与实践; Project number: 407) from Research and Practice Project of Higher Education \& Pedagogy Reform in Henan Province in 2019 (in Chinese: 2019年度河南省高等教育教学改革研 究与实践项目) and Xuchang Industrial Enterprise Pollution Control "Looking Back" Technical Services (in Chinese: 许昌市工业企业污染治理“回头看”技 术服务) from 2020 University-Government Cooperation Project (in Chinese: 2020年许昌市校地合作项目)

The funding bodies played no roles in the design of the study and collection, analysis, and interpretation of data and in writing the manuscript. The content is solely the responsibility of the author and does not necessarily represent the official views of the Henan Provincial Government and Xuchang Municipal Government in China.

Availability of data and materials

https://dataverse.harvard.edu/dataverse/harvard?q=Rozelle\%20Scott

\section{Declarations}

Ethics approval and consent to participate

Not applicable.

Consent for publication

Not applicable.

\section{Competing interests}

The author declared no potential conflict of interest with respect to the research, authorship and/or publication of this article.

\section{Author details}

'International Issues Center, Xuchang University, Xuchang, Road Bayi 88, Xuchang, Henan, China. ${ }^{2}$ Family Issues Center, Xuchang University, Road Bayi 88, Xuchang, Henan, China. ${ }^{3}$ School of Business, Xuchang University, Road Bayi 88, Xuchang, Henan, China.

Received: 27 October 2020 Accepted: 13 April 2021

Published online: 23 April 2021

\section{References}

1. Garland EJ. Rages and refusals. Managing the many faces of adolescent anxiety. Can Fam Physician. 2001;47:1023-30.

2. Zacharopoulou V, Tsironi M, Zyga S, et al. Depressive symptoms among adolescent students in greek high schools. Health Psychol Res. 2014;2(3): 1962. Published 2014 Oct 22. https://doi.org/10.4081/hpr.2014.1962.

3. Burgić-Radmanović M, Burgić S. Comorbidity in children and adolescent psychiatry. Psychiatr Danub. 2010;22(2):298-300. 
4. Brodbeck J, Abbott RA, Goodyer IM, Croudace TJ. General and specific components of depression and anxiety in an adolescent population. BMC Psychiatry. 2011;11:191. Published 2011 Dec 7. https://doi.org/10.1186/14 71-244X-11-191.

5. Maldonado L, Huang Y, Chen R, Kasen S, Cohen P, Chen H. Impact of early adolescent anxiety disorders on self-esteem development from adolescence to young adulthood. J Adolesc Health. 2013;53(2):287-92. https://doi.org/1 0.1016/j.jadohealth.2013.02.025.

6. Dick DM, Bernard M, Aliev F, et al. The role of socioregional factors in moderating genetic influences on early adolescent behavior problems and alcohol use. Alcohol Clin Exp Res. 2009;33(10):1739-48. https://doi.org/1 0.1111/j.1530-0277.2009.01011.x.

7. Piotrowska PJ, Stride CB, Rowe R. Social gradients in child and adolescent antisocial behavior: a systematic review protocol. Syst Rev. 2012;1:38. Published 2012 Aug 23. https://doi.org/10.1186/2046-4053-1-38.

8. Green JG, Keenan JK, Guzmán J, Vinnes S, Holt M, Comer JS. Teacher perspectives on indicators of adolescent social and emotional problems. Evid Based Pract Child Adolesc Ment Health. 2017;2(2):96-110. https://doi. org/10.1080/23794925.2017.1313099.

9. Anselmi L, Menezes AM, Hallal PC, et al. Socioeconomic changes and adolescent psychopathology in a Brazilian birth cohort study. J Adolesc Health. 2012;51(6 Suppl):S5-S10. https://doi.org/10.1016/j.jadohealth.2012.06.026.

10. Lansford JE, Deater-Deckard K, Dodge KA, Bates JE, Pettit GS. Ethnic differences in the link between physical discipline and later adolescent externalizing behaviors. J Child Psychol Psychiatry. 2004;45(4):801-12. https://doi.org/10.1111/j.1469-7610.2004.00273.x.

11. Daeem R, Mansbach-Kleinfeld I, Farbstein I, et al. Correlates of mental disorders among minority Arab adolescents in Israel: results from the Galilee Study. Isr J Health Policy Res. 2019;8(1):14. Published 2019 Jan 21. https:// doi.org/10.1186/s13584-018-0281-5.

12. Scholten S, Velten J, Neher T, Margraf J. Wealth, justice and freedom: objective and subjective measures predicting poor mental health in a study across eight countries. SSM Popul Health. 2017;27(3):639-48. https://doi. org/10.1016/j.ssmph.2017.07.010 PMID: 29349252; PMCID: PMC5769050.

13. Wang Z, Du W, Pang L, Zhang L, Chen G, Zheng X. Wealth inequality and mental disability among the chinese population: a population based study. Int J Environ Res Public Health. 2015;12(10):13104-17. https://doi.org/10.33 90/ijerph121013104 PMID: 26492258; PMCID: PMC4627019.

14. Lê-Scherban F, Brenner AB, Schoeni RF. Childhood family wealth and mental health in a national cohort of young adults. SSM Popul Health. 2016; 2:798-806. https://doi.org/10.1016/j.ssmph.2016.10.008 PMID: 28584861; PMCID: PMC5455782.

15. Kavanagh AM, Aitken Z, Krnjacki L, LaMontagne AD, Bentley R, Milner A. Mental health following acquisition of disability in adulthood--the impact of wealth. PLoS One. 2015;10(10):e0139708. https://doi.org/10.1371/journal. pone.0139708 PMID: 26444990; PMCID: PMC4596479.

16. Lachaud J, Hruschka DJ, Kaiser BN, Brewis A. Agricultural wealth better predicts mental wellbeing than market wealth among highly vulnerable households in Haiti: Evidence for the benefits of a multidimensional approach to poverty. Am J Hum Biol. 2020;32(2):e23328. https://doi.org/10.1 002/ajhb.23328 Epub 2019 Sep 12. PMID: 31512352; PMCID: PMC7577539.

17. Rozelle, Scott, 2016, "mental health in rural China: comparisons across provinces and among subgroups of children and adolescents", https://doi. org/10.7910/DVN/H5HBI6, Harvard Dataverse, V1, UNF:6:q5fN/ XI5mrj7nyYNz08Gbw== [fileUNF]. Published on 15 Aug 2016.

18. Liu H, Shi Y, Auden E, Rozelle S. Anxiety in rural chinese children and adolescents: comparisons across provinces and among subgroups. Int J Environ Res Public Health. 2018;15(10):2087. Published 2018 Sep 22. https:// doi.org/10.3390/ijerph15102087.

19. Alslman ET, Abu Baker N, Dalky H. Mood and anxiety disorders among adolescent students in Jordan. East Mediterr Health J. 2017;23(9):604-10. Published 2017 Nov 19. https://doi.org/10.26719/2017.23.9.604.

20. Wang Z, Zheng X, Zhang L, Chen G. Changes in prevalence and socioeconomic factors of psychiatric disability among children in china from 1987-2006: a population based survey. Int J Environ Res Public Health. 2017; 14(3):279. Published 2017 Mar 9. https://doi.org/10.3390/ijerph14030279.

21. Park HY, Heo J, Subramanian SV, Kawachi I, Oh J. Socioeconomic inequalities in adolescent depression in South Korea: a multilevel analysis. PLoS One. 2012;7(10):e47025. https://doi.org/10.1371/journal.pone.0047025.

22. Qu Y, Jiang H, Zhang N, Wang D, Guo L. Prevalence of mental disorders in 6-16-year-old students in Sichuan province, China. Int J Environ Res Public
Health. 2015;12(5):5090-107. Published 2015 May 13. https://doi.org/10.33 90/ijerph120505090.

23. Lopes CS, Abreu Gde A, dos Santos DF, et al. ERICA: prevalence of common mental disorders in Brazilian adolescents. Rev Saude Publica. 2016;50(Suppl 1):14s. https://doi.org/10.1590/S01518-8787.2016050006690.

24. Yao YS, Kang YW, Jin YL, et al. A prevalence survey on the mental health of left behind adolescent in Anhui Province. Zhonghua Liu Xing Bing Xue Za Zhi. 2010;31(12):1359-62.

25. Al-Sughayr AM, Ferwana MS. Prevalence of mental disorders among high school students in National Guard Housing, Riyadh, Saudi Arabia. J Family Community Med. 2012;19(1):47-51. https://doi.org/10.4103/2230-8229.94015.

26. Lawrence D, Hafekost J, Johnson SE, et al. Key findings from the second Australian child and adolescent survey of mental health and wellbeing. Aust N Z J Psychiatry. 2016;50(9):876-86. https://doi.org/10.1177/000486741561 7836.

27. Kalmar S. A magyar gyermek- és ifjúságpszichiátria fejlődésének legfontosabb akadályai [Major obstacles in the development of child and adolescent psychiatry in Hungary]. Neuropsychopharmacol Hung. 2016; 18(2):93-109.

28. Lesinskiene S, Girdzijauskiene S, Gintiliene G, et al. Epidemiological study of child and adolescent psychiatric disorders in Lithuania. BMC Public Health. 2018;18(1):548. Published 2018 Apr 24. https://doi.org/10.1186/s12889-0185436-3.

29. Bhola P, Kapur M. Child and adolescent psychiatric epidemiology in India. Indian J Psychiatry. 2003;45(4):208-17.

30. Balistreri KS, Alvira-Hammond M. Adverse childhood experiences, family functioning and adolescent health and emotional well-being. Public Health. 2016;132:72-8. https://doi.org/10.1016/j.puhe.2015.10.034.

31. Diener $\mathrm{E}, \mathrm{Ng} \mathrm{W}$, Harter J, Arora R. Wealth and happiness across the world: material prosperity predicts life evaluation, whereas psychosocial prosperity predicts positive feeling. J Pers Soc Psychol. 2010;99(1):52-61. https://doi. org/10.1037/a0018066 PMID: 20565185.

32. He Z, Cheng Z, Bishwajit G, Zou D. Wealth inequality as a predictor of subjective health, happiness and life satisfaction among nepalese women. Int J Environ Res Public Health. 2018;15(12):2836. https://doi.org/10.3390/ ijerph15122836 PMID: 30545142; PMCID: PMC6313399.

33. Ng P, Chan KF, Ho WC. A study on mental health of secondary school students in three metropolitan cities in China: Hong Kong, Shanghai, and Beijing. Int J Adolesc Med Health. 2008;20(1):53-62. https://doi.org/10.1515/ ijamh.2008.20.1.53 PMID: 18540284.

34. Zhou L, Fan J, Du Y. Cross-sectional study on the relationship between life events and mental health of secondary school students in Shanghai, China. Shanghai Arch Psychiatry. 2012;24(3):162-71. https://doi.org/10.3969/j.issn.1 002-0829.2012.03.006 PMID: 25324621; PMCID: PMC4198848.

35. Mao ZH, Zhao XD. The effects of social connections on self-rated physical and mental health among internal migrant and local adolescents in Shanghai, China. BMC Public Health. 2012;12:97. https://doi.org/10.1186/14 71-2458-12-97 PMID: 22299776; PMCID: PMC3305514.

36. Zhang Y, Zhang D, Li X, Ip P, Ho F, Jiang Y, et al. Daily time-use patterns and obesity and mental health among primary school students in shanghai: a population-based cross-sectional study. Sci Rep. 2017;7(1):16200. https:// doi.org/10.1038/s41598-017-15102-4 PMID: 29170506; PMCID: PMC5700943.

37. Chen $\mathrm{Q}$, Chen $\mathrm{Y}$, Zhao Q. Impacts of boarding on primary school students' mental health outcomes - instrumental-variable evidence from rural northwestern China. Econ Hum Biol. 2020;39:100920. https://doi.org/10.101 6/j.ehb.2020.100920 Epub 2020 Aug 14. PMID: 32919377.

38. Tang B, Wang Y, Gao Y, Wu S, Li H, Chen Y, et al. The effect of boarding on the mental health of primary school students in Western Rural China. Int J Environ Res Public Health. 2020;17(21):8200. https://doi.org/10.3390/ijerph1 7218200 PMID: 33172019; PMCID: PMC7664204.

39. Davies J, Sullivan S, Zammit S. Adverse life outcomes associated with adolescent psychotic experiences and depressive symptoms. Soc Psychiatry Psychiatr Epidemiol. 2018;53(5):497-507. https://doi.org/10.1007/s00127-01 8-1496-z.

40. Savolainen J, Eisman A, Mason WA, Schwartz JA, Miettunen J, Järvelin MR. Socioeconomic disadvantage and psychological deficits: pathways from early cumulative risk to late-adolescent criminal conviction. J Adolesc. 2018; 65:16-24. https://doi.org/10.1016/j.adolescence.2018.02.010.

41. Green JG, McLaughlin KA, Alegría M, et al. School mental health resources and adolescent mental health service use. J Am Acad Child Adolesc Psychiatry. 2013;52(5):501-10. https://doi.org/10.1016/j.jaac.2013.03.002. 
42. De Los RA, Augenstein TM, Wang M, et al. The validity of the multiinformant approach to assessing child and adolescent mental health. Psychol Bull. 2015;141(4):858-900. https://doi.org/10.1037/a0038498.

43. Almirall D, Chronis-Tuscano A. Adaptive interventions in child and adolescent mental health. J Clin Child Adolesc Psychol. 2016;45(4):383-95. https://doi.org/10.1080/15374416.2016.1152555.

44. Skokauskas N, Fung D, Flaherty LT, et al. Shaping the future of child and adolescent psychiatry. Child Adolesc Psychiatry Ment Health. 2019;13:19. Published 2019 Apr 11. https://doi.org/10.1186/s13034-019-0279-y.

45. Moulton V, Goodman A, Nasim B, Ploubidis GB, Gambaro L. Parental wealth and children's cognitive ability, mental, and physical health: evidence from the UK millennium cohort study. Child Dev. 2020. https://doi.org/10.1111/ cdev.13413 Epub ahead of print. PMID: 32939765.

46. Weinberg D, Stevens GWJM, Duinhof EL, Finkenauer C. Adolescent socioeconomic status and mental health inequalities in the Netherlands, 2001-2017. Int J Environ Res Public Health. 2019;16(19):3605 Published 2019 Sep 26. https://doi.org/10.3390/ijerph16193605.

47. McLaughlin KA, Costello EJ, Leblanc W, Sampson NA, Kessler RC Socioeconomic status and adolescent mental disorders. Am J Public Health. 2012;102(9):1742-50. https://doi.org/10.2105/AJPH.2011.300477.

48. Cheng J, Wang RC, Yin X, Fu L, Liu ZK. U-shaped relationship between years of residence and negative mental health outcomes among rural-to-urban children in migrant schools in beijing, china: the moderating effects of socioeconomic factors. Front Public Health. 2017;5:168. Published 2017 Aug 2. https://doi.org/10.3389/fpubh.2017.00168.

49. Qidwai W, Ishaque S, Shah S, Rahim M. Adolescent lifestyle and behaviour: survey from a developing country. PLoS One. 2010;5(9):e12914. Published 2010 Sep 27. https://doi.org/10.1371/journal.pone.0012914.

50. Morgan JK, Shaw DS, Forbes EE. Physiological and behavioral engagement in social contexts as predictors of adolescent depressive symptoms. J Youth Adolesc. 2013;42(8):1117-27. https://doi.org/10.1007/s10964-012-9815-2.

\section{Publisher's Note}

Springer Nature remains neutral with regard to jurisdictional claims in published maps and institutional affiliations.

Ready to submit your research? Choose BMC and benefit from:

- fast, convenient online submission

- thorough peer review by experienced researchers in your field

- rapid publication on acceptance

- support for research data, including large and complex data types

- gold Open Access which fosters wider collaboration and increased citations

- maximum visibility for your research: over $100 \mathrm{M}$ website views per year

At $\mathrm{BMC}$, research is always in progress.

Learn more biomedcentral.com/submissions 